\title{
FINITE COVERS OF 3-MANIFOLDS CONTAINING ESSENTIAL SURFACES OF EULER CHARACTERISTIC $=0$
}

\author{
SADAYOSHI KOJIMA
}

(Communicated by Haynes R. Miller)

\begin{abstract}
We give a short proof and a slight generalization of a theorem of John Luecke, that a compact connected orientable irreducible 3-manifold containing an essential torus is finitely covered by a torus bundle or manifolds with unbounded first Betti numbers.
\end{abstract}

1. Introduction. It has been proved by Luecke in his thesis that a compact orientable irreducible 3-manifold containing an essential torus is finitely covered by either a torus bundle or manifolds with unbounded first Betti numbers (see [3]). The purpose of this paper is to reprove this result and to extend it to manifolds containing essential annuli.

The argument of Luecke is based on the geometric structure which is the thesis of the uniformization theory developed by Jaco-Shalen, Johannson and Thurston. Our argument is based not on the uniformization theory but rather its topological consequence, that is, the residual finiteness of Haken manifold groups. Since we start from this fact, the argument becomes relatively short. Also we have a little advantage to extend the result for manifolds containing essential annuli. After some preliminaries, we will give our argument in the last section.

I am grateful to John Luecke for sending me a copy of [3], which contains a nice exposition of the motivation of that and hence this work.

2. Preliminaries. Throughout this paper, $M$ denotes a 3 -manifold and $\Pi$ the fundamental group of $M$. We refer to Hempel's book [1] for the definitions of standard terminology in 3-manifold topology. We say a properly embedded surface $S \subset M$ is essential if it is incompressible and not boundary parallel.

We now prepare a few lemmas which will be used later.

LEMMA 1. (1) $M$ contains a two-sided nonseparating surface if and only if $b_{1}(M)>0$.

(2) If $M$ contains disjoint two-sided surfaces $S_{1}$ and $S_{2}$ so that $M-\left(S_{1} \cup S_{2}\right)$ is connected, then $M$ is finitely covered by manifolds with unbounded $b_{1}$.

Proof. (1) is obvious. The assumption of (2) is equivalent to $\Pi$ having a representation onto a free group of rank two. A free group of rank two contains free subgroup of finite index and arbitrarily large rank. Thus $\Pi$ contains a subgroup of finite index which has a representation onto a free group of arbitrary rank. Since the rank of the target gives a lower bound on $b_{1}$ of the finite cover of $M$ associated to this subgroup, we are done.

Received by the editors April 24, 1986.

1980 Mathematics Subject Classification (1985 Revision). Primary 57M05, 57M10.

Key words and phrases. 3-manifold, essential surface, residual finiteness. 
LEMMA 2. Let $G$ be a group. Suppose $G$ admits a tower of normal subgroups of finite index: $G=G_{0}>G_{1}>\cdots$ so that $\bigcap_{p=0}^{\infty} G_{p}=\{e\}$ and so that $G / G_{p}$ is abelian for all $p \geq 0$. Then $G$ is abelian.

Proof. The abelianization defines the onto homomorphism $h: G \rightarrow H_{1}(G)$. It is enough to show that $h$ is injective. Take a nontrivial element $\alpha$ of $G$. Since $\alpha$ is nontrivial, there is some $p \geq 1$ so that $G_{p}$ does not contain $\alpha$. Since $G / G_{p}$ is abelian, the projection: $G \rightarrow G / G_{p}$ passes through the universal abelian group $H_{1}(G)$. This means that $\alpha$ is mapped by $h$ to a nontrivial element. Since $\alpha$ was arbitrary, $h$ becomes injective.

LEMMA 3. Let $M$ be a compact connected orientable irreducible 3-manifold with nonempty boundary. If $M$ has an infinite abelian fundamental group, then $M$ is homeomorphic to either $S^{1} \times D^{2}$ or $T^{2} \times I$.

PROOF. There is a complete list of abelian 3-manifold groups; see for instance [1]. Then by a standard method in 3-manifold theory, we get the conclusion.

Here is one consequence of the 3-dimensional uniformization theory. It is originally due to Thurston [4] and a detailed argument was presented by Hempel [2].

THEOREM 4. The fundamental group of a Haken manifold is residually finite.

3. Proof. Let us state the theorem to be proved.

THEOREM. Let $M$ be a compact connected orientable irreducible 3-manifold containing an essential surface homeomorphic to the torus or the annulus. Then $M$ is finitely covered by either a torus bundle over the circle, $T^{2} \times I$, or manifolds with unbounded first Betti numbers.

The proof occupies the rest of the paper. We divide the argument into two parts according to whether an essential surface is separating or nonseparating.

We first deal with a separating surface. Suppose that $M$ contains a separating essential surface, $S$, homeomorphic to the torus or the annulus. $S$ splits $M$ into two pieces $U$ and $V$. We use the notation: $\pi_{1}(U)=A, \pi_{1}(V)=B$ and $\pi_{1}(S)=\Gamma$.

Proposition 5. Let $S \subset M$ be as above. Then there is a finite covering $\pi: \tilde{M} \rightarrow M$ so that a component of $\pi^{-1}(S)$ is nonseparating in $\tilde{M}$.

Here is an easy criterion of the existence of such a covering.

LEMMA 6. Suppose that there is a normal subgroup $\Delta<\Pi$ of finite index satisfying

(i) $[A: A \cap \Delta]>[\Gamma: \Gamma \cap \Delta]$ and

(ii) $[B: B \cap \Delta]>[\Gamma: \Gamma \cap \Delta]$.

Then the covering associated to $\Delta$ has the property in Proposition 5.

ProOF. Take the covering $\pi: \tilde{M} \rightarrow M$ associated to $\Delta$. Condition (i) shows that the restriction of $\pi^{-1}(S)$ to a component of $\pi^{-1}(U)$ is not connected. Condition (ii) shows that the same is true for $V$. Since the covering is regular, the result follows by easy combinatorics.

PROOF OF PROPOSITION 5. Assuming that $\Pi$ has no subgroups of finite index having the property in Lemma 6 , we will prove this by reducing to the other easy cases for finding a covering we look for. 
Since $\Pi$ is residually finite by Theorem 4 , we take a descending sequence of normal subgroups of finite index: $\Pi=\Delta_{0}>\Delta_{1}>\Delta_{2}>\cdots$, so that $\bigcap_{p=0}^{\infty} \Delta_{p}=$ $\{e\}$. We first claim that $\left[A: A \cap \Delta_{p}\right] /\left[\Gamma: \Gamma \cap \Delta_{p}\right]$ is a nondecreasing function on $p$. To see this, take the cover $\tilde{M}$ associated to $\Delta_{p}$. Then this ratio can be identified with the number of components of the restriction of $\pi^{-1}(S)$ to a connected component of $\pi^{-1}(U)$ in $\tilde{M}$. Since $\left\{\Delta_{p}\right\}$ defines a tower of coverings, it cannot decrease. The same is true for $B$.

Thus since we assumed that there are no subgroups of $\Pi$ of finite index having the property in Lemma 6 , we have two cases.

(a) $\left[A: A \cap \Delta_{p}\right]=\left[\Gamma: \Gamma \cap \Delta_{p}\right]$ for all $p \geq 1$, or

(b) $\left[B: B \cap \Delta_{p}\right]=\left[\Gamma: \Gamma \cap \Delta_{p}\right]$ for all $p \geq 1$.

These two are symmetric and we may assume by switching the roles of $U$ and $V$ if necessary that (a) is the case.

Condition (a) shows that the homomorphism: $\Gamma / \Gamma \cap \Delta_{p} \rightarrow A / A \cap \Delta_{p}$ induced by the inclusion is an isomorphism. Since $\Gamma$ is abelian, $A$ is abelian by Lemma 2 . Since it is a fundamental group of a compact irreducible 3-manifold with nonempty boundary, $U$ is homeomorphic to either $S^{1} \times D^{2}$ or $T^{2} \times I$ by Lemma 3 . Since $S \subset \partial U$ was incompressible and not $\partial$-parallel in $M, S$ cannot be homeomorphic to the torus. We have already finished the proof when $S$ is homeomorphic to the torus.

Hereafter suppose that $S$ is homeomorphic to the annulus. Apart from the above argument for a while, we first deal with the case when both $U$ and $V$ are homeomorphic to $S^{1} \times D^{2}$. Let $n$ and $m$ be the indices of $\Gamma \cong \mathbf{Z}$ in $A \cong \mathbf{Z}$ and $B \cong \mathbf{Z}$ respectively. Both are $\geq 2$ since $S$ is not $\partial$-parallel. Then there is an $n m /(n, m)$-fold covering $\pi: \tilde{M} \rightarrow M$ to which $S$ lifts. $\tilde{M}$ consists of $m /(n, m)$ copies of the connected $n$-fold cover of $U$ and $n /(n, m)$ copies of the connected $m$-fold cover of $V . \pi$ is the covering we are looking for in this case.

We next suppose that $U$ is homeomorphic to $S^{1} \times D^{2}$ but $V$ is not. Let $n$ be the index of $\Gamma$ in $A$. $n \geq 2$ since $S$ is not $\partial$-parallel. Take an $n$-fold cyclic cover of $M$ which consists of $n$ copies of $V$ and the connected $n$-fold cover of $U$. Denote this cover by $M^{\prime}$ and a component of the lifts of $S$ by $S^{\prime} . S^{\prime}$ splits $M^{\prime}$ into two pieces $U^{\prime}$ and $V^{\prime}$. Neither $U^{\prime}$ nor $V^{\prime}$ is homeomorphic to $S^{1} \times D^{2}$. Furthermore, since $\Pi$ admits no subgroups of finite index having the property in Lemma 6 , neither does $\Pi^{\prime}=\pi_{1}\left(M^{\prime}\right)$ with respect to $S^{\prime}$. Letting $U^{\prime}=U$ and $V^{\prime}=V$, we have reduced to the case when neither $U$ nor $V$ is homeomorphic to $S^{1} \times D^{2}$. The same reduction works also when $V$ is homeomorphic to $S^{1} \times D^{2}$ but $U$ is not. Thus that is the only case we must deal with in the rest.

Now go back to the argument at the top, which says that the real remaining case is only when $U$ is homeomorphic to $T^{2} \times I$ and $V$ is not homeomorphic to $S^{1} \times D^{2}$. Then take a double cover $M^{\prime}$ of $M$ which consists of two copies of $V$ and a connected double cover of $U$. Let $S^{\prime}$ be a component of the preimage of $S$. $S^{\prime}$ splits $M^{\prime}$ into two pieces $U^{\prime}$ and $V^{\prime}$, where we let $V^{\prime}$ be a component of the preimage of $V$. Neither $U^{\prime}$ nor $V^{\prime}$ is homeomorphic to $S^{1} \times D^{2}$. Using the argument above, we may assume that $A^{\prime}=\pi_{1}\left(U^{\prime}\right)$ or $B^{\prime}=\pi_{1}\left(V^{\prime}\right)$ is abelian. If $A^{\prime}$ is so, then $U^{\prime}$ is homeomorphic to $T^{2} \times I$ and hence $V$ must be homeomorphic to $S^{1} \times D^{2}$. This is not the case. If $B^{\prime}$ is abelian, then $V^{\prime}$ is homeomorphic to $T^{2} \times I$ and $M$ becomes 
a union of two copies of $T^{2} \times I$ along the annuli on the boundary. $M$ in this case obviously has a covering having the property in Proposition 5.

We next deal with a nonseparating surface. Suppose that $M$ contains a nonseparating essential surface $S$ homeomorphic to either the torus or the annulus. We use the notations: $\overline{M-S}=W$ and $\pi_{1}(W)=A, \pi_{1}(S)=\Gamma$. We denote two copies of $S$ on $\partial W$ by $S$ and $S^{\prime}$. If $W$ is homeomorphic to $S \times I$ so that $S$ is identified with $S \times\{0\}$, then $M$ fibers over the circle with $S$ as a fiber. We will be concerned with the other case.

PROPOSITION 7. Suppose that $S$ is not a fiber. Then there is a finite covering $\pi: \tilde{M} \rightarrow M$ so that $\pi^{-1}(S)$ contains two components $S_{1}$ and $S_{2}$ with $\tilde{M}-\left(S_{1} \cup S_{2}\right)$ being connected.

We start again $\cdot$ with an easy criterion.

LEMMA 8. Suppose that there is a normal subgroup $\Delta<\Pi$ of finite index so that $[A: A \cap \Delta]>[\Gamma: \Gamma \cap \Delta]$. Then the covering associated to $\Delta$ has the property in Proposition 7.

PROOF. Take the covering $\pi: \tilde{M} \rightarrow M$ associated to $\Delta$. $\pi$ induces a covering: $\tilde{W} \rightarrow W$. The condition on the index means that the preimage of $S \subset W$ to a component of $\tilde{W}$ is not connected. Take any two of the components and let $S_{1}$ and $S_{2}$ be the corresponding surfaces in $\tilde{M}$. Then they obviously have the property in Proposition 7.

ProOF OF PROPOSITION 7. Assuming that $\Pi$ has no subgroups of finite index having the property in Lemma 8 , we will prove the proposition by reducing to cases for which it is easy to find the appropriate cover.

Since $\Pi$ is residually finite by Theorem 2.4 , we take a descending sequence of normal subgroups of finite index: $\Pi=\Delta_{0}>\Delta_{1}>\Delta_{2}>\cdots$, so that $\bigcap_{p=0}^{\infty} \Delta_{p}=$ $\{e\}$. We have by assumption that $\left[A: A \cap \Delta_{p}\right]=\left[\Gamma: \Gamma \cap \Delta_{p}\right]$ for all $p \geq 0$. This condition on the index means that the injective homomorphism $\Gamma / \Gamma \cap \Delta_{p} \rightarrow$ $A / A \cap \Delta_{p}$ is an isomorphism for all $p$. Since $\Gamma / \Gamma \cap \Delta_{p}$ is abelian for all $p, A$ is abelian by Lemma 2 . Hence $W$ is homeomorphic to $S^{1} \times D^{2}$ or $T^{2} \times I$ by Lemma 3. Again we have already finished the proof when $S$ is homeomorphic to the torus since this is the case we excluded.

Hereafter suppose that $S$ is homeomorphic to the annulus. We first furthermore suppose that $W$ is homeomorphic to $S^{1} \times D^{2}$. Let $n$ be the index of $\Gamma$ in $A$. Then $n \geq 2$ since $S$ is not $\partial$-parallel. Take the connected $n$-fold cover of $W$, to which $S$ and $S^{\prime}$ lift. This extends to an $n$-fold covering of $M$ having the property in Proposition 7.

Suppose that $W$ is homeomorphic to $T^{2} \times I$. Let $H$ be the subgroup of $A$ generated by the images of $\pi_{1}(S)$ and $\pi_{1}\left(S^{\prime}\right)$ by the inclusions. If $H$ is not all of $A$, then there is a subgroup of $A$ of finite index containing $H$. The associated cover of $W$ to this subgroup extends to a finite cover of $M$ to which $S$ lifts. In particular, it has the property in Proposition 7. If $H=A$, then take the subgroup of $A$ which consists of all squares. This defines a 4-fold cover of $W$ in which the preimage of $S$ and that of $S^{\prime}$ both have two components. It also extends to a cover of $M$ which has the property in Proposition 7. 
PROOF OF THE THEOREM. Suppose that $M$ contains a separating essential surface homeomorphic to the torus or the annulus. By Proposition 5, there is a finite cover of $M$ which contains a nonseparating essential surface of the same topological type. We thus start from $M$ that contains a nonseparating surface. Then by Proposition 7 , there is a finite cover $\tilde{M}$ of $M$ containing two disjoint surfaces $S_{1}$ and $S_{2}$ so that $\tilde{M}-\left(S_{1} \cup S_{2}\right)$ is connected unless $M$ is $T^{2} \times I$ or a torus bundle. The Theorem now follows from Lemma 1.

\section{REFERENCES}

1. J. Hempel, 3-manifolds, Ann. of Math. Studies, no. 86, Princeton Univ. Press, Princeton, N.J., 1976.

2. __ Residual finiteness for 3-manifolds, Combinatorial Group Theory and Topology, edited by S. Gersten and J. Stallings, Ann. of Math. Studies, no. 111, Princeton Univ. Press, Princeton, N. J., 1987, pp. 379-396.

3. J. Luecke, Finite covers of 3-manifolds containing essential tori, preprint (1986).

4. W. Thurston, Three dimensional manifolds, Kleinian groups and hyperbolic geometry, Bull. Amer. Math. Soc. 6 (1982), 357-381.

Department of Mathematics, Tokyo Metropolitan University, Fukasawa, SETAGaya, TOKYO, JAPAN 48109

Current address: Department of Mathematics, University of Michigan, Ann Arbor, Michigan 